\title{
Author Index Vol. 46, 1994
}

Ackermann, H. 70 Arndt, H.J. 265 Aucouturier, J ,-L. 188

Barber Watson, J. 214 Bijleveld,H. 250 Bonet, M. 27 Brown, W.S., Jr. 64

Carballo,G. 171 Casan,P. 27 Clegg,R.T. 180

Delong, V. 188 Dongen, H. van 250

Felton,R.H. 109

Gentil,M. 188 Gregory, H.H. 213

Haapanen, M.-L. 57 Häge,A. 241,298 Hartelius,L. 9 Heaton, J.M. 180 Hertrich,I. 70 Hirose,H. 139

Johannsen, H.S. 241,298

Kammer, H. 35,42 Kiese-Himmel, C. 288 Koch, A. 35,42 Kruse,E. 288 Kuhr,A. 232

Laukkanen, A.-M. 86 Lebrun,Y. 250 Leclercq, V. 127 Lees, R.M. 223 LilaTrujillo,P. 123

Luelsdorff, P.A. 271,281

Mans, E.J. 1 Marjatta Jaroma, S. 193 Mendoza, E. 171 Miller, D.G. 157 Milutinovic, Z. 135

Morris, R.J. 64

Niedzielska, G. 115 Niimi,S. 139

O'Leary,I.K. 180

Parker, A.J. 180 Pesák,J. 97 Piérart,B. 127 Preus,A. 254 Pruszewicz, A. 115

Qvarnström, M.J. 193

Reuter,W. 35,42 Rommel, D. 241,298

Sambuis,E. 188 Schäfer,A. 265 Schönweiler, R. 18 Schulze,H. 241,298 Schutte,H.K. 157 Scotto

Di Carlo, N. 79 Svec,J. 97 Svensson, P. 9 Swidziński, P. 115

Tellervo Laine, M. 193 Tsunoda,K. 139

Vandevelde, I. 127

309 